\title{
DESENVOLVIMENTO DE UM DISPOSITIVO DE ELETROFIAÇÃO AUTOMATIZADO PARA TESTES DE PRECISÃO
}

\author{
A. L. S. M. REUTER ${ }^{1}$, A. P. S. IMMICH ${ }^{1}$ \\ ${ }^{1}$ Departamento de Engenharias, Universidade Federal de Santa Catarina, Campus de \\ Blumenau - Blumenau. \\ E-mail para contato: andre.reuter@hotmail.com
}

\begin{abstract}
RESUMO - Eletrofiação é um processo eletrostático muito popular para produção de não tecidos e nanofibras. Atualmente existem diversas pesquisas que utilizam equipamento de eletrofiação, no entanto, existe uma carência no desenvolvimento deste equipamento, fazendo com que as máquinas disponíveis no mercado não tenham necessariamente o padrão de confiabilidade necessário. Dessa forma, o objetivo desse projeto é a construção de uma máquina de eletrofiação automatizada, de baixo custo que atenda especificações de controle de temperatura e umidade. Para construção da máquina, os seguintes componentes serão projetados: bomba infusora, fonte de tensão, plataforma coletora giratória e eixo de fiação móvel para eletrofiação em dois níveis, horizontal e vertical. Com a construção deste equipamento de eletrofiação automatizado, espera-se realizar experiências e provas de conceito, com maior precisão e confiabilidade necessárias para o respaldo científico.
\end{abstract}

\section{INTRODUÇÃO}

No processo de eletrofiação, uma solução polimérica é submetida a um campo elétrico. A solução permanece na extremidade de um tubo capilar e ação do campo elétrico induz cargas a solução. A repulsão das cargas causa uma força diretamente oposta a tensão superficial do líquido. Com intensidade suficiente do campo elétrico, a superfície do líquido na extremidade do capilar se alonga para uma superfície cônica conhecida como cone de Taylor. Quando a força de repulsão das cargas supera a tensão superficial do líquido, um jato eletricamente carregado da solução é ejetado do cone de Taylor. O solvente da solução evapora e a fibra polimérica carregada é depositada aleatoriamente sobre um coletor metálico (Reneker e Doshi, 1995). Eventualmente, toda a solução polimérica na extremidade do capilar é processada, então para haver continuidade no processo é necessário fluxo de solução para o capilar. Para isso usa-se uma bomba infusora, capaz de fornecer vazões extremamente pequenas.

Primeiramente descrita por Formhals (1938), a técnica de eletrofiação foi aplicada em diversos setores industriais para o desenvolvimento de nanofibras e nanofios ao longo dos anos. Entretanto, até a década de 2000, não houve grandes avanços em direção a automação desse processo assim como estudos sobre as variáveis de controle que são essenciais para a produção de fibras de qualidade. Como pode-se notar existem algumas variáveis cruciais. $\mathrm{O}$ comportamento e a estabilidade do campo elétrico, a interação do fluido com o campo 
elétrico, além de propriedades fluídicas como viscosidade e tensão superficial, vazão da solução para o capilar, distância entre a extremidade do capilar e a base coletora, e variáveis ambientais como temperatura, umidade e fluxo de ar através do jato (Doshi e Reneker, 1995). Apesar de cruciais, muitas das variáveis citadas não possuem controle apropriado nos equipamentos atuais e alguns procedimentos (como posicionamento do capilar) ainda são realizados manualmente, ainda que exista desenvolvimento na área de controle como modelos do efeito da uniformidade da distribuição do campo elétrico em eletrofiação (Yang et al., 2008), criação de novos tipos de capilares para redução de instabilidade do jato (Lee et al., 2014) e desenvolvimento de sistemas totalmente automatizados (Endler et al., 2015).

Atualmente existem diversas pesquisas que utilizam equipamento de eletrofiação, no entanto, existe uma carência no desenvolvimento do próprio aparato de eletrofiação, fazendo com que as máquinas disponíveis no mercado não tenham necessariamente o padrão de confiabilidade necessário. Além disso, o custo de aquisição de uma máquina de eletrofiação muitas vezes não está ao alcance de muitos pesquisadores e centros de pesquisa. Dessa forma, o objetivo desse projeto é a construção de uma máquina de eletrofiação automatizada, de baixo custo que atenda especificações de controle de temperatura e umidade. A máquina deverá ser capaz de executar o processo de eletrofiação em dois eixos distintos (horizontal e vertical) e contar com uma base coletora giratória. Para este projeto a fonte de alta-tensão necessária para geração do campo elétrico e a bomba infusora também serão construídas.

\section{MATERIAIS E MÉTODOS}

O protótipo proposto consiste na montagem de todos os elementos envolvidos no processo de eletrofiação, de forma que possam ter acionamento automático e controle de variáveis ambientais, fazendo com que a interferência do usuário seja reduzida. Todos os componentes mecânicos estruturais foram projetados utilizando o software SolidWorks 2016 SP4. As peças poliméricas foram fabricadas por uma impressora 3D Prusa Air 2 desenvolvida na Universidade Federal de Santa Catarina. Utilizou-se essencialmente o Nylon (Taulman Alloy 910) para as peças que integram a plataforma de eletrofiação e o poli(ácido láctico) para as peças que compõe a bomba infusora. Um serviço terceirizado de corte a laser foi utilizado para fabricação da peça estrutural da plataforma de eletrofiação em acrílico. Os componentes metálicos e eletrônicos foram adquiridos individualmente para montagem da bomba infusora, do gerador de tensão, da placa coletora, bem como do gabinete da máquina. Os conjuntos de movimentação da plataforma e da bomba infusora são idênticos e utilizam motor de passo NEMA 17 A1740CHNBK-CS, 200 passos/revolução, de 3,5 kgf.cm. A transmissão para movimento linear é feita utilizando um conjunto de fuso trapezoidal/castanha TR8, passo 8 mm. Ainda constituindo o sistema de movimentação temos eixos retificados de aço inoxidável 304 de 8 mm de diâmetro com bucha polimérica Igus GFM-0810-15.

O controlador utilizado é uma placa de desenvolvimento Arduino MEGA 2560 com processador Atmel 2560 com clock de $16 \mathrm{MHz}$. Para controle dos motores utilizou-se um driver A4988. A programação é feita em linguagem C++ e o Arduino IDE é utilizado como ambiente de programação.

\section{RESULTADOS}


A seguir temos as etapas que compõe o projeto e construção do protótipo e a montagem dos componentes citados acima.

\subsection{Plataforma de eletrofiação e gabinete da máquina}

O projeto da máquina foi concebido inicialmente pela plataforma de eletrofiação, sendo a parte principal do equipamento, onde os capilares de infusão e a base coletora são posicionados. Na Figura 1.a temos a disposição de todos os componentes. A peça móvel central da plataforma aloja os capilares e possui os eletrodos de cobre para contato elétrico. $\mathrm{O}$ objetivo desse módulo é ser o mais compacto possível para que a rotação de todo o conjunto em $90^{\circ}$ seja possível através da peça de pivô, que é vista na Figura 1.a.

Figura 1 - Projeto da plataforma: a) Imagem da disposição dos componentes; b) Imagem real da plataforma montada.
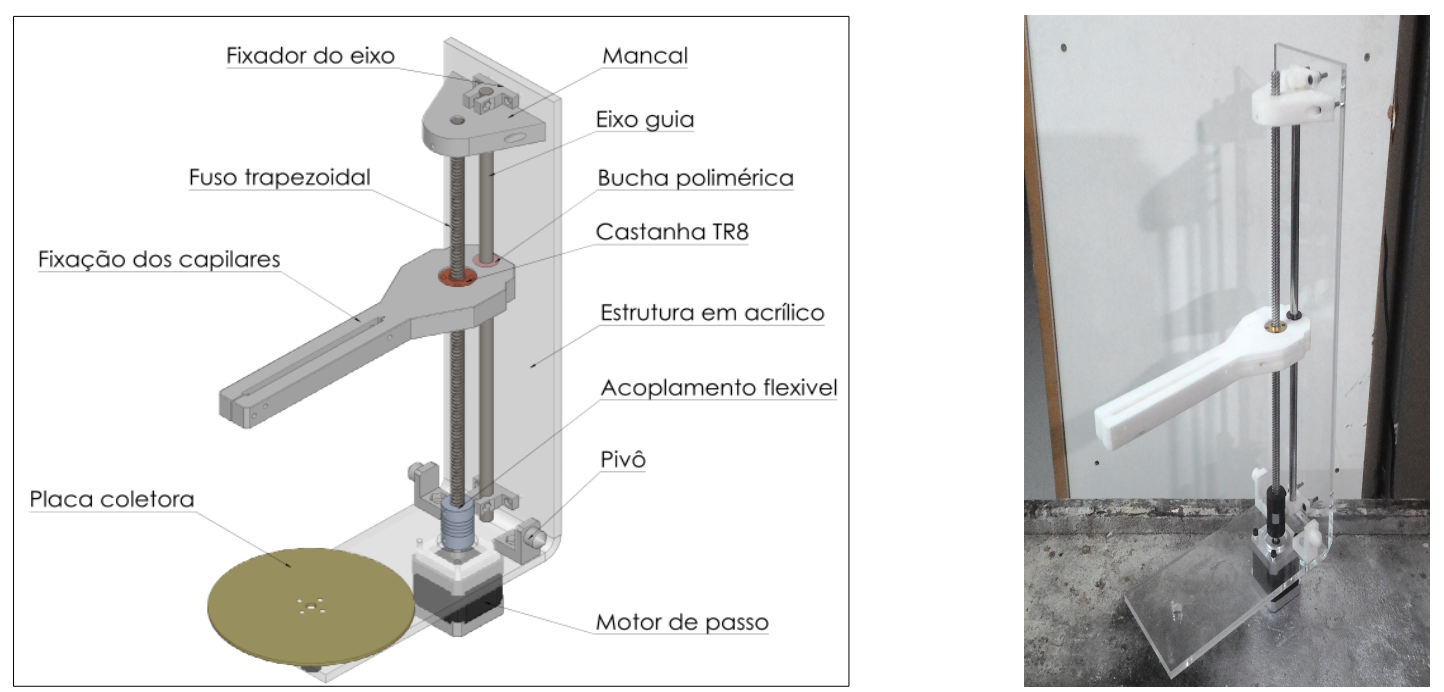

O motor de passo é utilizado pela excelente acurácia desse tipo de motor, pela possibilidade do controle de posição em malha aberta, e o alcance de velocidades e acelerações elevadas (podendo chegar a $150 \mathrm{~mm} / \mathrm{s}$ a uma aceleração de $3000 \mathrm{~mm} / \mathrm{s}^{2}$ ) fazendo com que o posicionamento do capilar seja rápido e preciso. O torque estático do motor assegura que a peça central de fixação dos capilares não se moverá durante o processo. $\mathrm{O}$ material das peças na cor branca na Figura 1.b é o Taulman Alloy 910, um tipo de nylon para impressão em 3D que conta com as mesmas propriedades da poliamida $6 / 6.9$, além da baixa reatividade com outros materiais fazendo com que seja ideal para uso em ambientes com agressão leve de vapores de solventes. A placa coletora é giratória sendo movimentada por um micromotor DC fixado na parte inferior da plataforma. Uma peça de acoplamento faz a fixação da placa ao motor possibilitando o uso de diferentes diâmetros do coletor. Toda plataforma é fixada na base do gabinete da máquina onde é posicionado a segunda parte do conjunto de pivô que possibilita uma rotação limitada em $90^{\circ}$, assim é possível obter o processo eletrofiação em dois eixos distintos. Com a plataforma posicionada na vertical, outros processos podem ser executados como a eletroaspersão, por exemplo.

O gabinete da máquina aloja todos os componentes incluindo a interface com o usuário. É uma estrutura feita em aço inoxidável e pintura epóxi para isolamento elétrico e proteção 
contra corrosão. O gabinete possui perfurações para instalação de microventiladores que compõem um sistema de controle de temperatura do ambiente de eletrofiação. A temperatura e a umidade são fatores de influência direta na qualidade final do processo de eletrofiação, pois interferem no comportamento do campo elétrico e nas propriedades fluídicas da solução polimérica.

\subsection{Bomba infusora}

A bomba infusora projetada possui capacidade para duas seringas, fornecendo uma vazão mínima de $0.5 \mathrm{~mL} / \mathrm{h}$. O sistema motriz é idêntico ao da plataforma de eletrofiação. A Figura 2 mostra o projeto concluído. A bomba possui todas as peças impressas em 3D utilizando poli (ácido latico) (PLA). Apesar de o PLA ser um dos polímeros mais utilizados em eletrofiação para produção de não tecidos e nanofibras, este também é um excelente polímero para pequenas estruturas e peças funcionais devido a suas características mecânicas e a densidade do material. A bomba infusora é posicionada no exterior do gabinete da máquina sendo protegida dos solventes. Além disso, a bomba fica protegida de ruídos eletrostáticos, que podem alterar os sinais enviados ao motor prejudicando a precisão da vazão entregue pela bomba.

Figura 2 - Bomba infusora projetada e montada para o equipamento de eletrofiação.

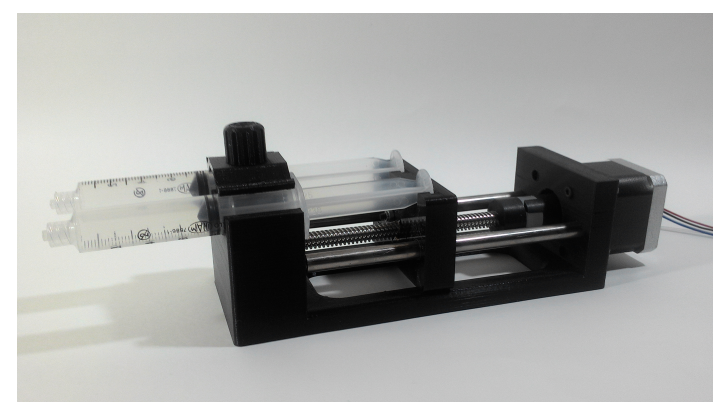

\subsection{Fonte de alta-tensão}

A fonte de alta-tensão é o componente do equipamento de eletrofiação de maior custo comercial, principalmente devido a questões de segurança envolvidas na fabricação e no uso do equipamento, além da dificuldade de manter a precisão e qualidade da tensão de saída da fonte. A tensão desejada é de $40 \mathrm{kV}$ com corrente não superior a $1 \mathrm{~mA}$. O projeto da fonte utiliza como componente principal um indutor flyback, um tipo de transformador de núcleo saturável utilizado principalmente em circuitos para excitação de raios catódicos ou formação de arcos voltaicos para geração de laser. Em resumo, este componente é capaz de aumentar a tensão que lhe é aplicada, sendo mais compacto que um transformador comum. Para usar este componente é necessário um driver. Essencialmente, é um circuito que entrega uma tensão senoidal em alta frequência (acima de $10 \mathrm{MHz}$ ). Existem diversas opções para esse circuito, a escolhida para o projeto é uma variação do clássico oscilador de Royer. De acordo com Royer e Bright (1957) o oscilador é um circuito inversor de frequência (conhecido como conversor DC-AC) que usa elementos semicondutores (transistores) para ação de chaveamento de forma a receber uma tensão contínua e transformá-la em tensão alternada para uso em cargas eletromagnéticas (transformadores). O circuito utilizado é descrito por Baciu et al. (2015). Normalmente nesse tipo de circuito os transistores usados são MOSFETs, que são 
consideravelmente mais eficientes que os bipolares (TBJ). No entanto, o uso de MOSFETs, exige grande precisão e sincronia no chaveamento dos gates para que o circuito comece a oscilar, diferentemente dos TBJ, que aceitam uma pequena diferença na sincronização do chaveamento das bases, garantindo a oscilação.

A tensão de saída é controlada com a tensão de entrada, sendo diretamente proporcional até uma região de saturação (por volta de $64 \mathrm{~V}$ na entrada) devido as especificações dos componentes. A frequência de ressonância do circuito LC (indutor capacitor) define a frequência de chaveamento dos transistores e consequentemente a frequência da tensão entregue ao "primário" do indutor flyback. Este circuito é capaz de entregar altas frequências com estabilidade garantindo um funcionamento estável do flyback. O consumo de corrente do circuito com 12 VDC na entrada chega a $2 \mathrm{~A}$ com o secundário aberto, caindo para $1.75 \mathrm{~A}$ com o secundário em curto. Com 12 VDC na entrada a tensão na saída chega a aproximadamente $10 \mathrm{kV}$. O flyback utilizado possui internamente um circuito retificador, por isso a tensão de saída da fonte é contínua. Não foi possível medir a tensão de ripple da saída, mas os resultados de simulações feitas em LTSpice IV indicam menos de $50 \mathrm{~V}$. A corrente na saída ainda não foi estimada e em simulações não passa de $10 \mathrm{~mA} \mathrm{em} 10 \mathrm{kV}$.

\subsection{Controle e interface}

O controle de todas as variáveis é unificado em uma única interface, diferentemente de algumas máquinas comerciais que possuem interfaces independentes para a bomba infusora e a fonte de alta-tensão. Isso facilita o trabalho do usuário e mantém a programação da máquina mais simples.

Essencialmente a programação se resume no controle dos motores de passo do sistema, a variação da tensão da fonte, e a interface com o usuário, além das tarefas em segundo plano, consistindo no controle de temperatura e as verificações de segurança. O controle dos motores de passo é feito em malha aberta devido ao alto custo de encoders para esse tipo de aplicação, e pela precisão e repetibilidade que os motores de passo apresentam. O controle é feito utilizando um driver A4988 que utiliza a técnica de microstepping criada para redução do ruído de vibração quando o motor opera em baixas velocidades e que aumenta a resolução que o motor pode atingir. No software para controle é utilizada a biblioteca AccelStepper, uma interface orientada a objeto que utiliza a geração de perfis de velocidade e aceleração descritas por Austin (2004) e que permite a utilização de velocidades extremamente baixas, que são utilizadas no motor da bomba infusora.

Para o controle de tensão um conversor buck é utilizado para a variação da tensão de entrada da fonte. Visto que a tensão de saída varia linearmente com a tensão de entrada nos pontos de operação previstos, a lei de controle conta apenas com um controlador proporcional. O tempo de resposta e as dinâmicas de regime transiente são muito rápidas em relação a dinâmica total do processo e não possuem influencia na resposta da fonte. Outra malha de controle é a de temperatura do ambiente em que o processo ocorre. Visto que o gabinete da máquina é fechado, o controle de temperatura utiliza microventiladores e termistores em diferentes regiões do gabinete. O controlador utilizado é do tipo on-off, visto que o processo libera pouco ou nenhum calor no ambiente e este controlador serve apenas para garantir determinada temperatura durante o processo. 


\section{CONCLUSÃO}

Ainda que não fora possível realizar os ensaios de eletrofiação dado que o protótipo se encontra em desenvolvimento, a construção da máquina nos forneceu diversas constatações. A concepção da plataforma de eletrofiação se mostrou uma boa opção ao design convencional, abrindo um leque de possibilidades para um único equipamento. Uma das dificuldades encontradas no projeto foi a integração das interfaces de controle das variáveis de eletrofiação (vazão, posição da plataforma e tensão do campo elétrico), isso exigiu o uso de algumas técnicas de programação e integração de hardware como o uso do protocolo de comunicação $\mathrm{I}^{2} \mathrm{C}$. No entanto, foi, de fato, possível realizar a integração necessária, mostrando que este tipo de equipamento pode ser controlado com uma plataforma open source de baixo custo, oferecendo maior possibilidade de melhorias em trabalhos futuros. Assim, levando em conta o exposto, as ferramentas utilizadas e a forma do projeto tornam a máquina uma plataforma para experiências e provas de conceito, com precisão e confiabilidade necessárias para respaldo científico, contribuindo para maiores avanços na área de eletrofiação.

\section{REFERÊNCIAS}

AUSTIN, D. Generate stepper-motor speed profiles in real time. 2014 Disponivel em: $<$ http://www.embedded.com/design/mcus-processors-and-socs/4006438/Generatestepper-motor-speed-profiles-in-real-time> Acesso em: 20 de abr. 2017

BACIU, I.; CUNTAN, C. D.; FLORUTA, M. Self-oscillating inverter with bipolar transistors. International Conference on Applied Sciences. 2015.

DOSHI, J.; RENEKER, D. H. Electrospinning Process and Applications of Electrospun Fibers. Journal of Electrostatics., v. 35, p. 151-160, 1995

ENDLER, B.; DORY, R.; YUAN, T.; DeSILVA, M. Development of a Novel Electrospinning System with Automated Positioning and Control Software. San Antonio: Naval Medical Research Unit San Antonio, 2015

FORMHALS, A. Artificial fiber construction U.S. Patent No. 1,975,504. 15 ago. 1936, 22 fev. 1938.

LEE, B.; JEON, S.; PARK, H.; LEE, G.; YANG, H.; YU, W. New Electrospinning Nozzle to Reduce Jet Instability and Its Application to Manufacture of Multi-layered Nanofibers. Scientific Reports, Nature. v. 4, ${ }^{\circ}$ artigo 6758, 2014

ROYER, G. H.; BRIGHT, R. L. ELECTRICAL INVERTER CIRCUITS U.S. Patent No. 2,783,384. 6 ago. 1954, 26 fev. 1957.

YANG, Y.; JIA, Z.; LIU, J.; LI, Q.; HOU, L.; WANG, L.; GUAN, Z. Effect of electric field distribution uniformity on electrospinning. Journal of Applied Physics. v. 103, 2008. 